\title{
Holographic Entanglement Entropy from Numerical Relativity
}

\section{Ecker*}

Institute for Theoretical Physics, Vienna University of Technology

Wiedner Hauptstr. 8-10/136, 1040 Vienna, Austria

E-mail: ecker@hep.itp.tuwien.ac.at

\section{S. A. Stricker}

Institute for Theoretical Physics, Vienna University of Technology

Wiedner Hauptstr. 8-10/136, 1040 Vienna, Austria

E-mail: stricker@hep.itp.tuwien.ac.at

In this proceedings we show the evolution and equilibration of nonlocal quantities such as equal time two-point functions for operators of large conformal weight and holographic entanglement entropy in $\mathscr{N}=4$ super Yang Mills theory using the gauge gravity duality. We use two different scenarios. One where the system is initially anisotropic and thermalizes and one which also includes inhomogeneities corresponding to colliding shocks on the gravity side.

Proceedings of the Corfu Summer Institute 2015 "School and Workshops on Elementary Particle Physics and Gravity"

1-27 September 2015

Corfu, Greece

${ }^{*}$ Speaker. 


\section{Introduction}

Understanding the complicated field dynamics taking place in a heavy ion collision is a great challenge to theorists. Comparing data from experiments at RHIC and the LHC to hydrodynamical simulations revealed that the produced state of matter behaves as a strongly coupled liquid with a very low shear viscosity over entropy ratio and extremal fast thermalization times. The nonperturbative nature of the expanding plasma calls for alternative methods to perturbation theory and lattice gauge theory. By now the gauge gravity duality has established itself as a valuable additional framework to study the dynamics of strongly coupled gauge theories. This duality maps a strongly coupled gauge theory in the limit of infinite colors to a supergravity theory in one dimension higher. In the context of heavy ion collisions usually the original form of the duality is used where asymptotically anti de Sitter space is mapped to $\mathscr{N}=4$ supersymmetric Yang-Mills (SYM) theory. Using SYM to gain insights into QCD can be justified by the fact that in the deconfined phase they share certain features such as a finite correlation length, no supersymmetry and the Wilson loop shows area law behavior. The thermalization process in the dual description is given in terms of gravitational collapse and black hole formation in asymptotically AdS spacetimes.

A crude gravitational dual of a heavy ion collision was first mimicked by the collision of two delta like gravitational shock waves [1]. Subsequently, the models were further refined by introducing anisotropy [2] and inhomogeneity [3]. By now the numerical algorithms have evolved to a point where simulations of off-central collisions of two localized lumps of matter, mimicking nucleus-nucleus and proton-nucleus collisions, are possible [4,5]. Due to the computational complexity in the aforementioned studies the canonical observables to monitor the evolution are the components of the local stress energy tensor.

The duality also offers a description to fairly easy obtain nonlocal quantities such as two-point functions, Wilson loops and entanglement entropy (EE) which on the gravity side permit a description in terms of invariant geometric objects such as geodesics, extremal surfaces and volumes [6]. These observables were first analyzed in collapsing shell models where a spherically symmetric shell made out of null dust collapses and forms a black hole [7, 8].

It turns out that the time evolution of the entanglement entropy in these models shows universal behavior and can be divided into three stages. The early quadratic initial growth is followed by a long linear growth and finally saturates. The quadratic and linear growth are independent from the entangling region, the dimension of the spacetime [9], the equation of state [10,11] and even for spacetimes with Lifshitz scaling and hyper scaling violation [12].

However, by changing the symmetries of the system the entanglement entropy can show different behavior and in this article we show the behavior of equal-time two point functions and entanglement entropy for geometries used to mimic heavy ion collisions, namely the homogeneous anisotropic case [13] and case of two colliding shockwaves [3].

\section{Theoretical setup}

In this section we summarize the theoretical foundations needed in rest of this article by introducing the geometry and concepts of how to compute equal time two-point functions and entanglement entropy holographically. 


\subsection{The geometry}

In the following we will study two cases imposing different symmetry assumptions. One where the spacetime is homogeneous but with an anisotropy present between the longitudinal and transverse coordinates with an $\mathscr{O}(2)$ symmetry in the transverse plane and one which is also inhomogeneous in the longitudinal direction, corresponding to the shock wave geometry. The ansatz for the line element that accounts for all these symmetries can be written conveniently in EddingtonFinkelstein coordinates

$$
\mathrm{d} s^{2}=-A \mathrm{~d} v^{2}+S^{2}\left(e^{-2 B} \mathrm{~d} y^{2}+e^{B} \mathrm{~d} \vec{x}^{2}\right)+2 \mathrm{~d} v(\mathrm{~d} r+F \mathrm{~d} y),
$$

where the functions $A, S, B$ and $F$ depend on the holographic coordinate $r$, (advanced) time $v$ and longitudinal coordinate $y$. In the homogeneous case the function $F=0$ and the other metric functions do not depend on $y$.

In order to obtain the geometry Einsteins equations have to be solved numerically for appropriate initial and boundary conditions.

\subsection{Two-point functions}

The equal time two-point functions for operators of large conformal weight $\Delta$ can be obtained from the length of space like geodesics via the formula

$$
\left\langle\mathscr{O}(t, \vec{x}) \mathscr{O}\left(t, \vec{x}^{\prime}\right)\right\rangle \approx \sum_{\text {geodesics }} e^{-\Delta L_{g}} \approx e^{-\Delta L},
$$

where to leading order in the geodesic approximation the sum over all geodesics is given by the one with the smallest length.

\subsection{Holographic entanglement entropy}

The holographic entanglement entropy of some region $A$ in the boundary can be computed by extremizing the area functional

$$
\mathscr{A}=\int \mathrm{d}^{3} \sigma \sqrt{\operatorname{det}\left(\frac{\partial X^{\mu}}{\partial \sigma^{a}} \frac{\partial X^{v}}{\partial \sigma^{b}} g_{\mu \nu}\right)}
$$

for a surface that extends into the bulk and ends on the boundary of the region $A$. It is conjectured that in the dual field theory the EE is given by

$$
S_{\mathrm{EE}}=\frac{\mathscr{A}}{4 G_{N}} .
$$

In the special case of stripe like regions boundary regions, with the finite directions chosen appropriately, the problem of finding geodesics can be reduced to finding geodesics in an auxiliary spacetime. 


\section{Isotropization}

Here we present results for the homogeneous and anisotropic situation where at initial time $t=0$ an anisotropy is introduced by choosing a particular form of the anisotropy function $B$ that only has support in the bulk

$$
B\left(r, v_{0}\right)=\frac{\beta}{r^{4}} \exp \left[-\left(\frac{1}{r}-\frac{1}{r_{0}}\right)^{2} / \omega^{2}\right]
$$

with $\beta=6.6, r_{0}=4$ and $\omega=1$ and then solving Einsteins equations numerically. The solution is summarized in Fig. 1 where on the 1.h.s. we show the most important features of the geometry and on the r.h.s. the evolution of transverse and longitudinal pressure. Note that, in the special case when the anisotropy function vanishes at the boundary, the energy density is constant. From this figure one can also see that the apparent horizon approaches the event horizon quickly and they start to coincide approximately at the same time when the system isotropizes. At late times the geometry approaches the Schwarzschild AdS geometry with a temperature given by $T=1 / \pi$ and the boundary coordinates are plotted in units of the temperature.

In asymptotically AdS spacetimes the length of geodesics diverges and a regularization scheme must be adopted. In order to make the approach to equilibrium most transparent we renormalize the geodesic length and EE in the following way

$$
\begin{aligned}
& L_{\mathrm{ren}}=\frac{L-L_{\mathrm{th}}}{L_{\mathrm{th}}} \\
& S_{\mathrm{ren}}=\frac{S-S_{\mathrm{th}}}{S_{\mathrm{th}}}
\end{aligned}
$$

where $L(S)$ is the unrenormalized length (EE) and $L_{\mathrm{th}}\left(S_{\mathrm{th}}\right)$ is the corresponding thermal value.
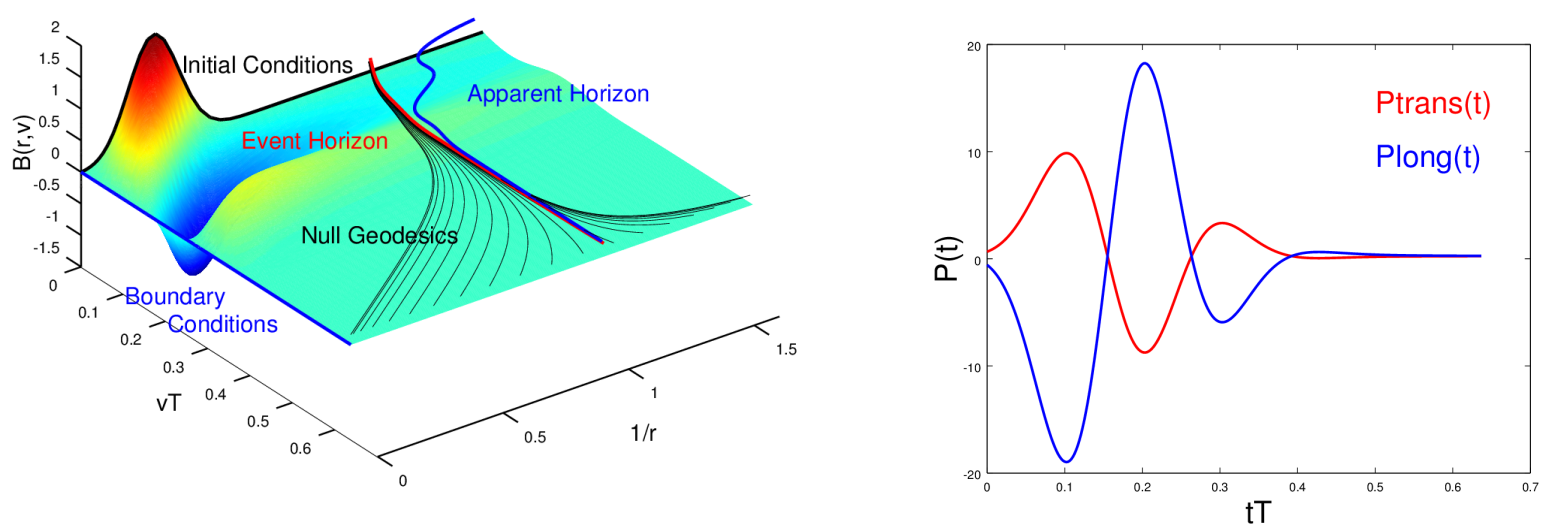

Figure 1: Left: anisotropy function $B(r, v)$. Right: transverse and longitudinal pressure. 


\subsection{Two point function}

We are now ready to present results for the equal time two-point functions but first we discuss the geometric shape of the geodesics. In collapsing shell models it was observed that in out-ofequilibrium situations space-like geodesics can actually reach beyond the apparent horizon and are able to probe regions which are not accessible to other observables and that a large fraction of the linear scaling originates from geodesics reaching beyond the apparent horizon.

At late times when the geometry is static the geodesics tend to move along the horizon but never cross it and for large enough boundary separations the EE approaches the thermal entropy as was shown in [6]. However, at early times when the system is far from equilibrium and the position of the apparent horizon changes with time the geodesics can reach beyond the horizon as is displayed in Fig. 2.
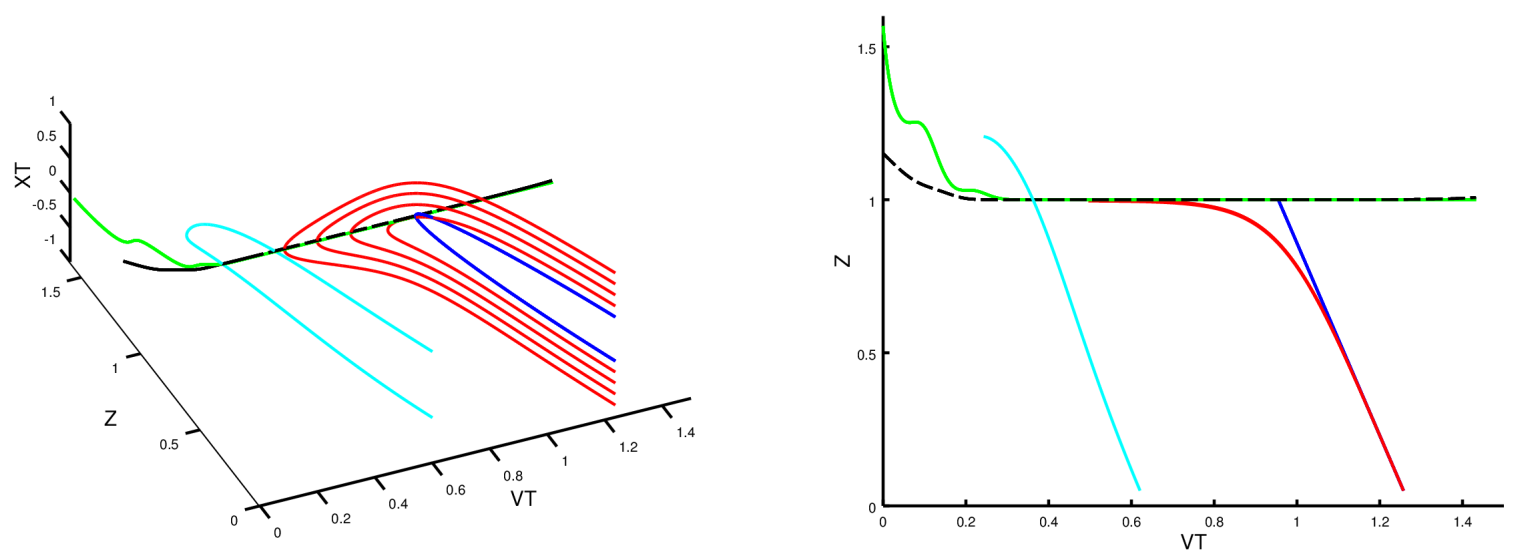

Figure 2: The green and black lines indicate the position of the apparent and event horizon, respectively. The blue curve is the Poincaré patch AdS geodesic we use to initialize the simulation; red curves are geodesics of different separation at late times which do not cross the horizon. The cyan curve in the left part of each plot is a geodesic which reaches beyond the horizon and probes the non-thermal region.

In Fig. 3 the evolution of the renormalized length for geodesics with boundary separations along the transverse and longitudinal direction is shown. The transverse and longitudinal components oscillate out of phase and this behavior is a manifestation of the pressure anisotropies visible in the evolution of the components of the stress energy tensor (see Fig. 1). For larger boundary separations the two-point functions approach their corresponding thermal value at later times, nicely showing the usual top-down thermalization pattern of strongly coupled theories. In addition the two-point functions thermalize later than the one-point function.

\subsection{Entanglement entropy}

For the holographic entanglement entropy the same qualitative features as for the two-point functions are observed as is shown in Fig. 4. Again, we observe out of phase oscillations for separations along the longitudinal and transverse direction. The EE reaches equilibrium later than the two-point function. The reason for the delay in equilibration can be understood geometrically. In 

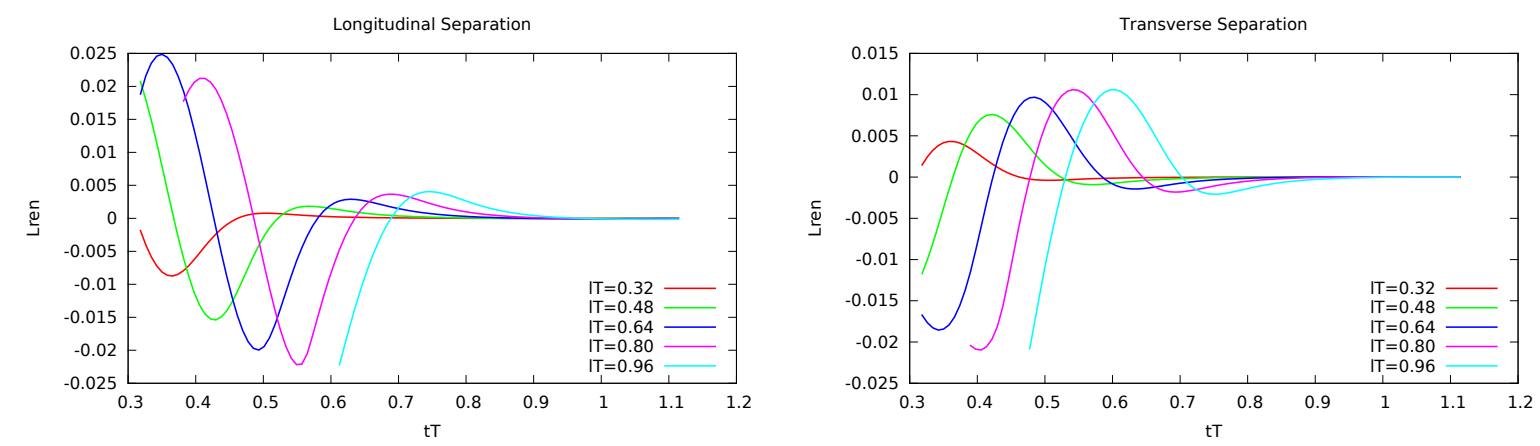

Figure 3: Renormalized length of geodesics for different separations in longitudinal and transverse directions.

order to obtain the EE we were able to reduce the problem of finding minimal surfaces to a problem of finding geodesics in an auxiliary spacetime. In this auxiliary spacetime the geodesics for the same boundary separation reach much deeper into the bulk and bend further back in time and therefore out-of-equilibrium effects influence their shape at later times. It might seem surprising that the EE can be become negative but this due the special setup we are considering. The energy momentum tensor is constant and the anisotropies in the line element change the length of geodesics out of phase. In the next section we will see that this oscillatory behavior can also be understood from a late time analysis.
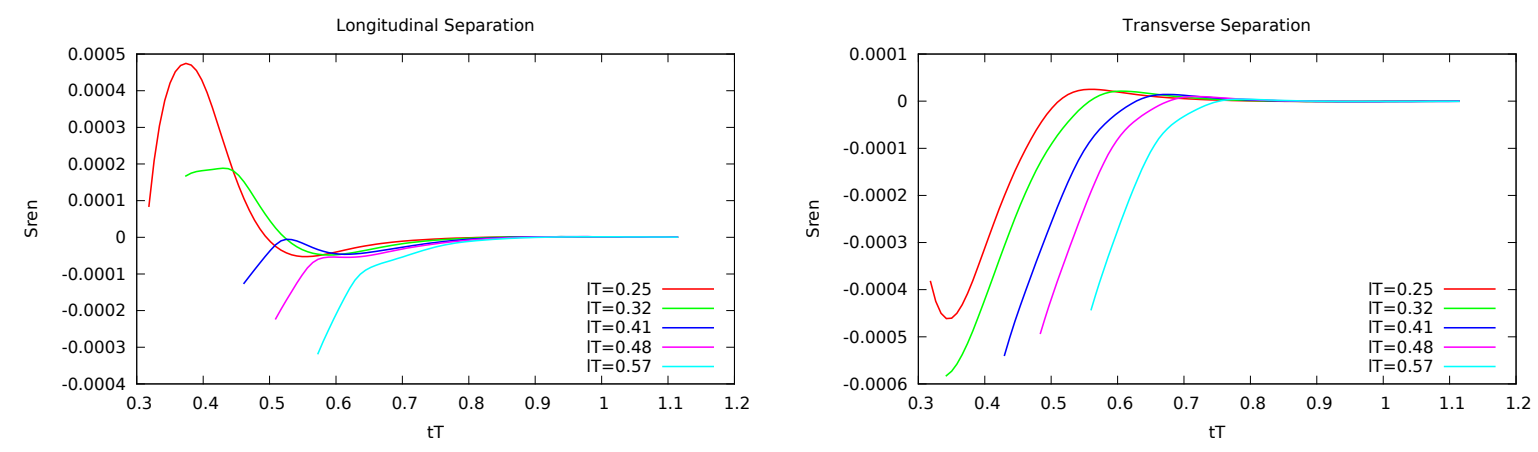

Figure 4: Longitudinal and transverse EE for different separations.

\subsection{Late time behavior and quasinormal modes}

At late times the geometry relaxes to the Schwarzschild black brane solution and one enters the linearized regime. As was observed already in [13, 14] the pressure anisotropies exhibit exponential damping and near equilibrium this damping is described by the lowest quasi normal mode which characterizes the response of the system to infinitesimal perturbations. To be more precise, the asymptotic form of the pressure anisotropies takes the form

$$
b_{4}(t) \sim \operatorname{Re}\left[c_{1} e^{-i \omega_{1} t}\right]
$$


where the lowest QNM obtained from the scalar channel of gravitational fluctuations is given by [15]

$$
\frac{\omega_{1}}{\pi T}= \pm 3.119452-2.746676 i
$$

We now demonstrate that at late times the approach to thermal equilibrium of the renormalized geodesic length and the EE is described by the lowest QNM. By multiplying the two observables with the imaginary part of the lowest QNM, $e^{\operatorname{Im}\left(\omega_{1} t\right)}$, we observe that after the initial far from equilibrium epoch the evolution of the observables is accurately described by the lowest QNM with constant amplitude and frequency. This behavior is shown in Fig. 5
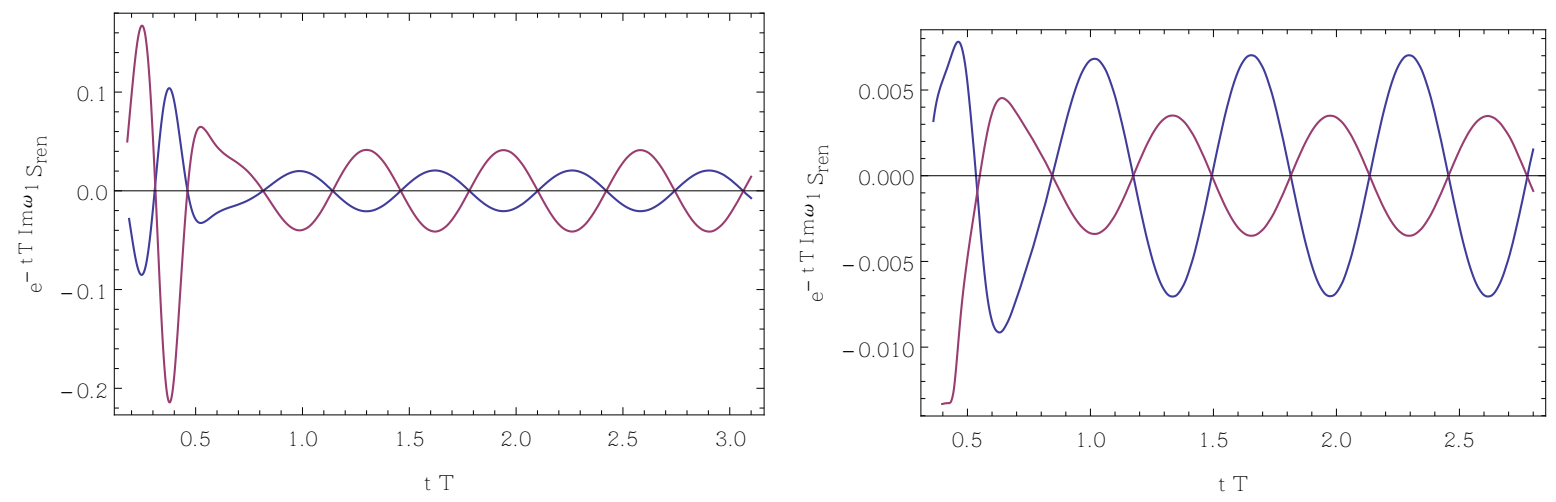

Figure 5: Left: Renormalized geodesic length for longitudinal (red) and transverse (blue) separation for $l T=0.32$ multiplied by the imaginary part of the lowest QNM. Right: Renormalized EE for the same parameters as on the left.

\section{Colliding shock waves}

Similarly to the previous section we can also obtain the renormalized geodesic length and EE in the colliding shock wave geometry where two sheets of energy with infinite extend in the transverse directions and finite extend in the longitudinal direction are used to mimic the collision of two highly Lorentz contracted nuclei. The two incoming shock waves are described by a Gaussian of width $\omega$ and amplitude $\mu^{3}$

$$
h(\tilde{t} \pm \tilde{y})=\frac{\mu^{3}}{\sqrt{2 \pi \omega^{2}}} e^{-\frac{(\tilde{z} \pm \tilde{y})^{2}}{2 \omega^{2}}} .
$$

where $\tilde{t}$ and $\tilde{y}$ are the time and longitudinal coordinates in Fefferman-Graham gauge, respectively. Depending on the thickness of the shocks two distinct scenarios appear. Wide shocks exhibit the so called full-stopping scenario. After the collision the shocks are slowed down, most of the energy density is concentrated in the central region and hydrodynamical explosion occurs. On the contrary, narrow shocks exhibit transparency. They almost pass through each other and although their shape gets altered they continue to move at the speed of light. In this work we take initial conditions corresponding to the wide shock scenario with width $\omega=0.5$. In Fig. 6 we display the evolution of the field theory energy density and longitudinal pressure in units of the energy density $\mu$ for our initial conditions. 

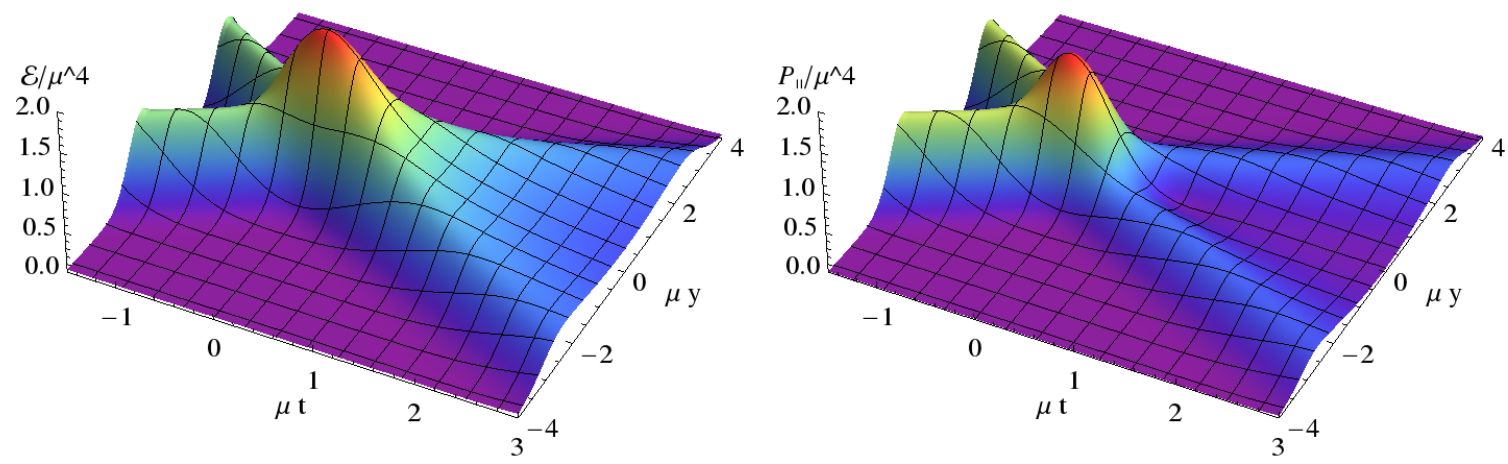

Figure 6: Energy density and longitudinal pressure as a function of time and longitudinal separation in units of $\mu$.

\subsection{Evolution of non-local observables}

We now present the time evolution of the renormalized geodesic length and EE for different boundary separations. In the case of colliding shock waves the system is also inhomogeneous and the calculation for the EE can only be reduced to finding geodesics in an auxiliary spacetime for separations along the longitudinal direction. In addition, at late times the geometry approaches the boost invariant geometry of perfect fluid dynamics and never relaxes to a static black hole. Therefore we renormalize the nonlocal observables by subtracting the corresponding pure AdS values

$$
\begin{array}{r}
L_{\mathrm{reg}}=\frac{L-L_{0}}{\Delta}, \\
S_{\mathrm{reg}}=4 G_{N}\left(\frac{S}{V}-\frac{S_{0}}{V_{0}}\right),
\end{array}
$$

where the geodesic length is given in units of the conformal scaling dimension Delta and the EE is divided by the corresponding infinite transversal stripe two-volumes $V$ and $V_{0}$.

The results are presented in Fig. 7. On the 1.h.s. the renormalized length is shown. Keeping in mind that the two-point-function is minus the exponential of the renormalized length (see equ. (2.2)) we observe the following. Depending on the separation, one starts with some non-vanishing initial correlations. For small separation the correlation is almost zero because at small distances the shocks have not interacted yet. As the shocks collide more and more short range correlations are destroyed and de-correlation happens in a linear fashion. After the collision correlations are restored due to new interactions during the collision.

On the r.h.s. of Fig. 7 the evolution of the EE is displayed. Before the collision the EE grows linearly, consistent with results from the collapsing shell models and reaches a maximum slightly after the energy density reaches its maximum at $\mu t=0$. At late times the EE shows polynomial fall off behavior. 
Compared to the isotropization case, for the wide shock geometry we did not find geodesics that extend beyond the horizon. However, for narrow shocks such geodesics exist and results will be presented elsewhere [16].
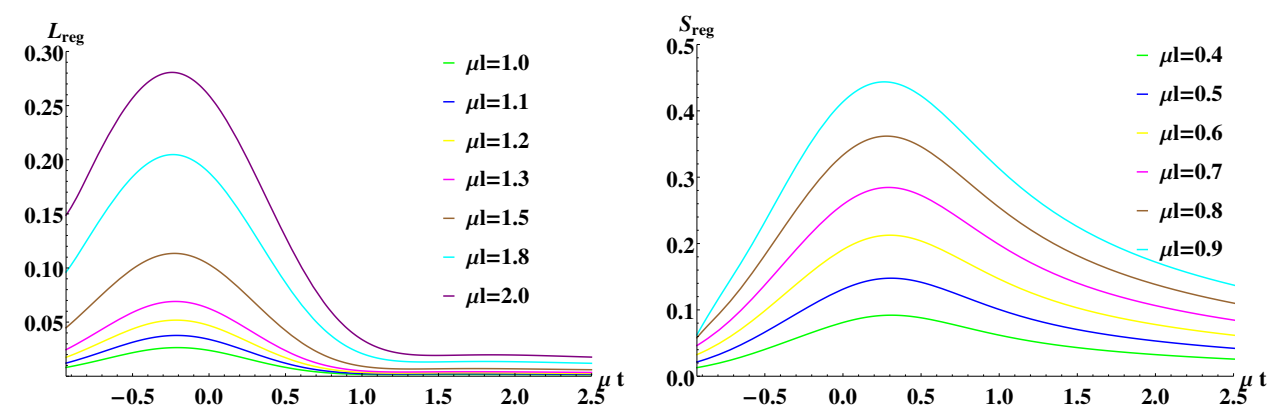

Figure 7: Left: Evolution of the renormalized geodesic length for different separations in units of $\mu$. Right: Evolution of the renormalized EE.

\section{Conclusions and Outlook}

In the paper at hand we showed the time evolution of non-local observables such as equal-time two point functions and entanglement entropy obtained via the holographic duality in geometries relevant for heavy ion collisions. Starting with a homogeneous but anisotropic set up we observed oscillatory behavior of both non-local observables with out of phase oscillations of the longitudinal and transverse directions. At late times the approach to thermal equilibrium is accurately described by the lowest lying quasinormal mode. In addition, if the geometry is sufficiently far away from equilibrium space like geodesics can extend beyond the apparent horizon and therefore we conclude that in highly dynamical systems these geodesics can be used to extract valuable information residing behind the apparent horizon not accessible to other probes. In the case of wide colliding shocks we found that the geodesics do not extend beyond the apparent horizon. The length of the geodesics and the EE show similar behavior. Both quantities increase before and decrease after the collision. The EE reaches the maximum value later and falls off more slowly.

In future work we plan to also study the influence of different initial conditions on the shape of nonlocal observables. It will be interesting to see if initial conditions leading to the transparency scenario manifest themselves in the shape of the curves. Another interesting extension of this work will be to study if the null energy condition is violated in the transparency scenario where the energy density can become negative in certain regions after the collision. If this is the case it will be a useful testing ground of the quantum null energy condition [17] and might provide further insights thereof.

\section{Acknowledgments}

The authors would like to thank Wilke van der Schee who provided us with the shock wave geometry necessary to carry out the calculation for the nonlocal observables. This work was sup- 
ported by the following projects of the Austrian Science Fund (FWF): P27182-N27, DKW1252N27. 


\section{References}

[1] D. Grumiller and P. Romatschke, On the collision of two shock waves in AdS(5), JHEP 08 (2008) 027 [0803.3226].

[2] P. M. Chesler and L. G. Yaffe, Horizon formation and far-from-equilibrium isotropization in supersymmetric Yang-Mills plasma, Phys.Rev.Lett. 102 (2009) 211601 [0812 . 2053].

[3] P. M. Chesler and L. G. Yaffe, Holography and colliding gravitational shock waves in asymptotically AdS 5 spacetime, Phys. Rev. Lett. 106 (2011) 021601 [1011.3562].

[4] P. M. Chesler and L. G. Yaffe, Holography and off-center collisions of localized shock waves, JHEP 10 (2015) 070 [1501.04644].

[5] P. M. Chesler, Colliding shock waves and hydrodynamics in small systems, Phys. Rev. Lett. 115 (2015), no. 24241602 [1506.02209].

[6] S. Ryu and T. Takayanagi, Holographic derivation of entanglement entropy from AdS/CFT, Phys.Rev.Lett. 96 (2006) 181602 [hep-th/ 0603001$].$

[7] J. Abajo-Arrastia, J. Aparicio and E. Lopez, Holographic Evolution of Entanglement Entropy, JHEP 1011 (2010) 149 [1006.4090].

[8] V. Balasubramanian, A. Bernamonti, J. de Boer, N. Copland, B. Craps et. al., Holographic Thermalization, Phys.Rev. D84 (2011) 026010 [1103.2683].

[9] H. Liu and S. J. Suh, Entanglement growth during thermalization in holographic systems, Phys.Rev. D89 (2014), no. 6066012 [1311.1200].

[10] V. Keranen, H. Nishimura, S. Stricker, O. Taanila and A. Vuorinen, Gravitational collapse of thin shells: Time evolution of the holographic entanglement entropy, 1502.01277.

[11] V. Keranen, H. Nishimura, S. Stricker, O. Taanila and A. Vuorinen, Dynamics of gravitational collapse and holographic entropy production, Phys.Rev. D90 (2014), no. 6064033 [14 05.7015 ].

[12] P. Fonda, L. Franti, V. Keränen, E. Keski-Vakkuri, L. Thorlacius et. al., Holographic thermalization with Lifshitz scaling and hyperscaling violation, JHEP 1408 (2014) 051 [1401.6088].

[13] M. P. Heller, D. Mateos, W. van der Schee and M. Triana, Holographic isotropization linearized, JHEP 1309 (2013) 026 [1304.5172].

[14] P. M. Chesler and L. G. Yaffe, Numerical solution of gravitational dynamics in asymptotically anti-de Sitter spacetimes, JHEP 1407 (2014) 086 [1309.1439].

[15] A. O. Starinets, Quasinormal modes of near extremal black branes, Phys. Rev. D66 (2002) 124013 [hep-th/0207133].

[16] C. Ecker, D. Grumiller, P. Stanzer, S. A. Stricker and W. van der Schee, Monitoring shock wave collisions with nonlocal observables, to appear.

[17] R. Bousso, Z. Fisher, J. Koeller, S. Leichenauer and A. C. Wall, Proof of the Quantum Null Energy Condition, Phys. Rev. D93 (2016), no. 2024017 [1509. 02542]. 